\title{
Autophagy Modulation in Disease Therapy: Where Do We Stand?
}

\author{
Michael P. Nelson • John J. Shacka
}

Published online: 1 October 2013

(c) Springer Science+Business Media New York 2013

\begin{abstract}
Since it was first described more than 50 years ago, autophagy has been examined in many contexts, from cell survival to pathogen sequestration and removal. In more recent years, our understanding of autophagy has developed sufficiently to allow effective targeted therapeutics to be developed against various diseases. The field of autophagy research is expanding rapidly, demonstrated by increases in both numbers of investigators in the field and the breadth of topics being addressed. Some diseases, such as the many types of cancer, have come to the fore in autophagy therapeutics research as a better understanding of their underlying mechanisms has surfaced. Numerous treatments are being developed and explored, from creative applications of the classic autophagy modulators chloroquine and rapamycin, to repurposing drugs approved for other treatments, such as astemizole, which is currently in use as an antimalarial and chronic rhinitis treatment. The landscape of autophagy modulation in disease therapy is rapidly changing and this review hopes to provide a cross section of the current state of the field.
\end{abstract}

Keywords Autophagy - Pharmacological therapy · Cancer · Ischemia-reperfusion · Stroke ·

Neurodegeneration · Neuropathology ·

Parkinson's disease · Alzheimer's disease .

Disease treatment

M. P. Nelson $(\bowtie) \cdot$ J. J. Shacka

Neuropathology Division, Department of Pathology, University of Alabama at Birmingham, Sparks Clinics, Room SC 930B, 1720 7th Ave S., Birmingham, AL 35294, USA e-mail: mpnelson@uab.edu

J. J. Shacka

Birmingham VA Medical Center, 700 19th St S., Birmingham, AL 35233, USA

\section{Introduction}

Autophagy, first known as focal cytoplasmic degradation, was first described as a cellular reaction to injury by cellular pathologists in the 1950s (reviewed in Oczypok et al.) [1]. After Christian de Duve coined the term "autophagy" in 1963, describing it as a cell death process, it has been a topic of interest to numerous investigators in multiple fields [2]. In the last 50 years or so, much has been discovered about autophagy, including the definition of distinct initiation mechanisms: macroautophagy for cytoplasm and organelles (hereafter referred to simply as "autophagy"), microautophagy for nonspecific degradation of proteins at the lysosomal membrane surface, and chaperone-mediated autophagy to target specific proteins [3-5]. Even specific processes governing the sequestration and degradation of mitochondria (mitophagy), lipids (lipophagy), and bacteria and other intracellular pathogens (xenophagy) have been identified [6-8].

Historically, both the methods used by investigators to study autophagy and the interpretations of results from those methods have suffered from a relative lack of standardization. In response to this and to the acceleration of the autophagy field, by corresponding with an increased number of investigators entering the field, a large cooperative effort was published in 2008 , followed by an update in 2012, defining guidelines for the use and interpretation of assays monitoring autophagy, hereafter referred to as "the guidelines" $[9,10 \bullet \cdot$. Some salient points from these reports follow, as they guide the interpretation of results from studies to be reviewed here. Autophagic flux is a dynamic process and is the accepted gold standard for investigating autophagy. It is defined in the guidelines as "...the entire process of autophagy including the delivery of cargo to lysosomes (via fusion of the latter with autophagosomes or amphisomes) and its subsequent breakdown 


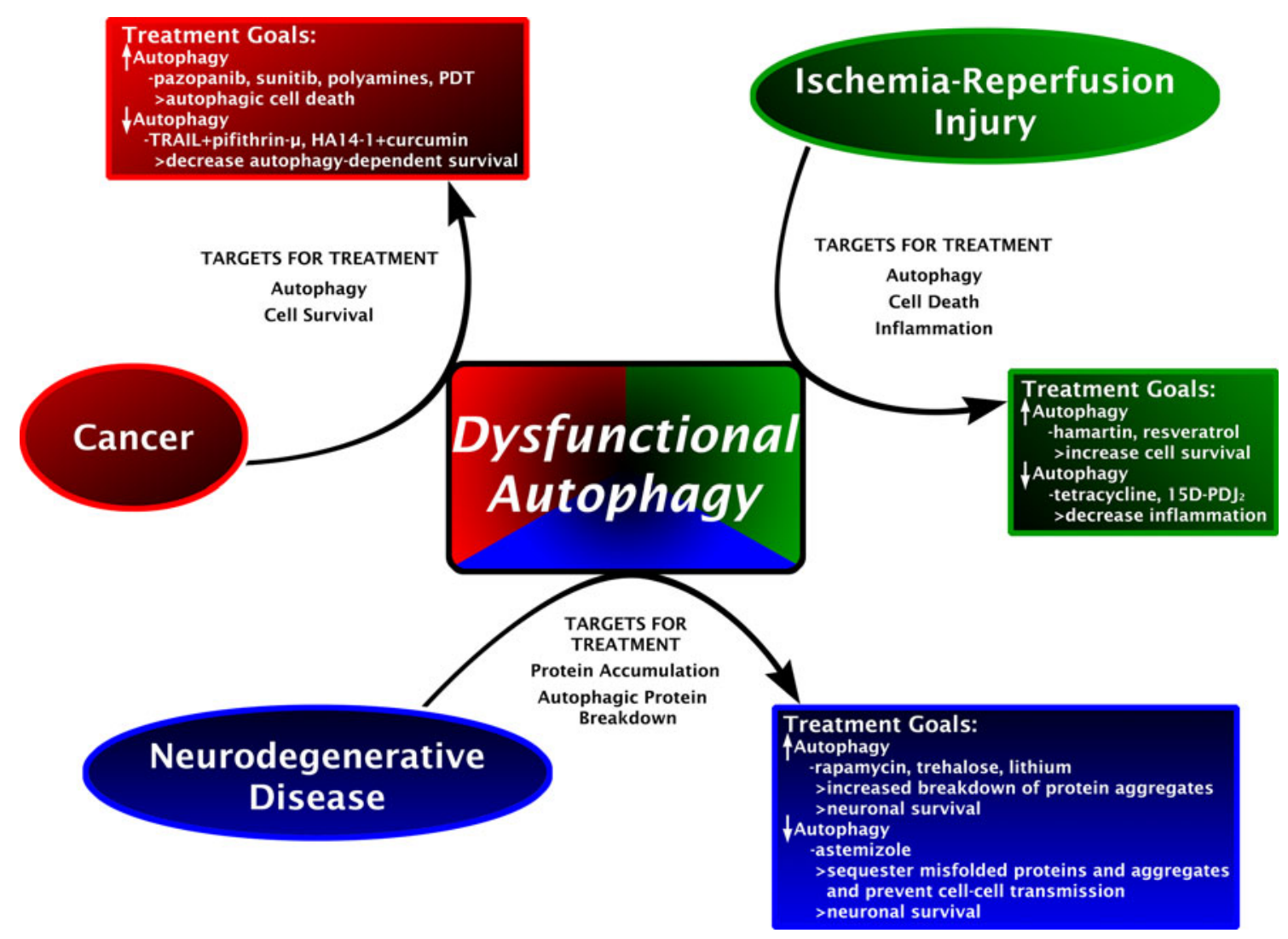

Fig. 1 Diagrammatic representation of the topics covered in this review. Dysfunctional autophagy results from and results in numerous disease states, including cancer, ischemia-reperfusion injury, and neurodegenerative diseases. This figure illustrates each disease state,

and release of the resulting macromolecules back into the cytosol (this may be referred to as productive or complete autophagy)" $[10 \bullet \cdot]$.

As the number of investigators studying autophagy has increased, the complexity of autophagy has become increasingly appreciated. Ordinarily, it is a homeostatic pathway for cell survival; however its dysfunction has been documented to also regulate cell death [11]. Conversely, the normal functioning of autophagy may also contribute to providing the energy necessary to initiate cell death [12]. Thus, cellular damage and death can result from autophagy being both too active and too inactive. Owing to its integral involvement in mechanisms of both cell death and survival, autophagy has become a highly sought-after drug target for a wide range of diseases, including many forms of cancer, stroke, neurodegenerative disease and immune disorders.

This review will explore current therapeutic strategies involving autophagy (Fig. 1). As a predominance of current research focuses on cancer, ischemia-reperfusion injury and neurodegenerative disease, we will be focusing on these disease states. Both activation and inhibition of autophagy are viable goals for drug treatment, and so they will each be discussed in the context of each disease state. the issues associated with autophagy, and treatment goals addressed by various pharmacological therapies, along with the expected result of those treatments (indicated by ">" symbols)

In addition, the integrated nature of lysosomal function to successful autophagic processes necessitates a discussion regarding therapies that target the lysosome. While it should not be taken as a comprehensive list, with this review we hope to provide a helpful cross section of the current state of autophagy modulation in disease therapy.

\section{Cancer}

The yin and yang of autophagy's effects on cell survival and impact on homeostasis result in some interesting findings regarding disease therapy. The treatment of cancer, in particular, highlights this tension. Some anti-cancer treatments are designed to enhance autophagy induction to promote cell death, while others are intended to decrease the cell survival-promoting mechanisms of autophagy to promote cancer cell death.

\section{Autophagy-Enhancing Cancer Therapy}

Some treatments that enhance autophagy are already in clinical use for cancer [13-15]. Among them are sunitinib 
and pazopanib, which are indicated for treatment of metastatic renal cell carcinoma [16, 17]. Both are tyrosine kinase inhibitors that target vascular endothelial growth factor receptors-1, -2, and -3 (VEGFR-1,-2,-3), but affect autophagy at different stages (Fig. 1). Pazopanib, in addition to its indication for metastatic renal carcinoma, is also used to treat soft tissue sarcoma [18]. As an oral treatment option, it has been shown to provide the benefit of progression-free survival [19]. Mechanistically, pazopanib increases autophagic flux, as measured by increased LC3-II conversion and decreased p62 accumulation, confirmed by treatment with the vacuolar-ATPase inhibitor bafilomycin A1 and time-dependent activation of ERK, leading to increased cell death that is markedly reversed by 3-MA treatment [20]. Pazopanib also induces cathepsin B activity that can mediate autophagic flux, and inhibition of cathepsin B by its specific inhibitor CA704-Me abrogates cell death [20, 21].

Although they are not yet in clinical use, synthetic polyamines are being explored for their putative toxicity to tumor cells [22]. Minarini et al. [22] examined the ability of symmetrically substituted long-chain polymethylene tetramines, methoctramine, and its analogs to kill HeLa cancer cells. They reported the ability of these polyamines to cause caspase-independent cell death via autophagy, since caspase activity was unchanged by treatment with the compound. Stating that these compounds are "powerful inducers of autophagy", the investigators showed significant increases in LC3-II conversion in HeLa cells concurrent with increased cell death, ultrastructural analysis showing organic material-containing vacuole accumulation in treated cells via electron microscopy, and acidic vesicle accumulation measured by acridine orange staining [22]. It is difficult, however, from this study to ascertain whether methoctramine and its analogs promote autophagy induction, as autophagic flux was not directly assessed, or if autophagy directly regulates cell death, as relative cell death was not assessed following inhibition of autophagy.

Another emerging cancer therapy involving autophagy induction is photodynamic therapy, a class of therapies that use electromagnetic radiation to activate drug molecules to generate reactive oxygen species in cancer cells. They are currently in use against various forms of cancer and have recently been shown to induce LC3-II conversion and vacuole accumulation, as measured by monodansylcadaverine (MDC) staining, both of which were reversible by wortmannin treatment and suggest autophagy induction [23]. The authors stated that this leads to autophagic rather than apoptotic cell death, as it is abrogated by wortmannin treatment and determined by MDC fluorescence intensity after treatment with the $\mathrm{LD}_{90}$ dose of PDT, which would normally cause death in $90 \%$ of treated cells. Mitophagyrelated cell death was ruled out by positive staining for intact mitochondrial membrane potential with tetramethylrhodamine, methyl ester. Autophagic cell death was further supported by an absence of apoptotic morphology through Hoechst nuclear staining [23-25].

\section{Autophagy-Inhibiting Cancer Therapy}

Many cancers are resistant to anti-cancer treatments, as is the case with apoptosis-inducing TRAIL treatment of human pancreatic cancer [26]. Because of this, some autophagy treatments are being sought to combat cancer drug resistance rather than to directly impact cancer cells. Pifithrin- $\alpha$ and $-\mu$ are known to modify p53 and so have potent effects on cell function. Pifithrin- $\mu$ (2-phenylethynesulfonamide) is also a small molecule inhibitor of inducible heat shock protein 70 (HSP70), which has been shown to bind and sequester cytosolic p53 [27]. Pifithrin- $\mu$ was shown to disrupt autophagy, as demonstrated by increased LC3-II conversion, LC3 puncta, increased autophagic vacuole size, and accumulation of p62 [26]. To support the autophagy dependence of pifithrin- $\mu$ enhancement on TRAIL-induced cell death, the authors showed that siRNA knockdown of Beclin-1 increased cell death by TRAIL and increased annexin V positivity [26-29]. As such, this disruption of autophagy is thought to contribute to the increased sensitivity of pancreatic cancer cells to TRAIL when the therapies are combined [26].

Another combination therapy being assessed for its potential is the effect of HA14-1, a competitive inhibitor of Bcl-2 that potently induces apoptosis, with that of curcumin, which is in the Indian spice turmeric. Treatment with HA14-1 plus curcumin has been shown to enhance autophagic cell death by an mTOR-dependent mechanism and through activation of ERK signaling, by examining autophagic flux through concentration- and time-dependent LC3-II conversion, LC3 puncta formation and 3-MAreversible cell death $[30,31]$. One study demonstrates that curcumin can be used in combination with HA14-1 to scavenge reactive oxygen species and limit collateral pathology due to HA14-1-induced apoptotic cell death [30].

\section{Lysosome Function Modification in Cancer}

Although tied together in the autophagy-lysosome pathway, the distinctions between autophagy and lysosomal activity allow for specific therapies geared toward modulation of the lysosome.

As indicated above, sunitinib is a potent anti-cancer treatment [32, 33]. Although it exhibits protein kinase inhibition mechanisms, it is distinct from the similar pazopanib in that it is lysosomotrophic [34]. At a physiological $\mathrm{pH}$, sunitinib freely crosses plasma membranes and so is 
allowed to accumulate in lysosomes where, due to the lower $\mathrm{pH}$, it is ionized and is unable to diffuse out. As a result of the accumulation of sunitinib, the lysosome swells in size, the $\mathrm{pH}$ is increased and so the functions of key lysosomal enzymes such as cathepsins are disrupted [35, 36]. Sunitinib also activates what the authors call "incomplete autophagy", measured by an increase in autophagic induction by time-dependent increase in LC3-II conversion but without the expected increase in p62 degradation [20]. This incomplete autophagy has been shown to lead to lysosomal-dependent necrosis, and so appears to be a mechanism of sunitinib's anti-cancer activity [20].

\section{Ischemia-Reperfusion Injury}

During stroke and other ischemic events, blood supply to the brain suddenly ceases. The enormous metabolic requirements of the brain combined with the lack of glycogen storage result in catastrophic effects when it is deprived of oxygen and nutrients. Neuronal cell death arises subsequent to loss of blood flow, and the effects of ischemic attacks can have long-term consequences in the form of collateral damage to surrounding cells and the activation of immune responses [37].

\section{Autophagy Enhancement in Ischemia-Reperfusion Injury}

Although the sudden depletion of oxygen and nutrients is putatively cytotoxic, many cell survival mechanisms are triggered in the interim, such as gene reprogramming, by modification of the metabolic pathway, ionic transport, and immune response genes after an initial ischemic attack, and metabolic downregulation [37]. Among them is autophagy and one inducer of it in the $\mathrm{Ca} 3$ region of the hippocampus during stroke is hamartin, a product of the tuberous sclerosis complex 1 gene (TSC1). Overexpression of hamartin induces autophagy through an mTORC1-dependent mechanism, increasing cell survival during ischemia, indicating that hamartin could be a drug target for increasing autophagy in the brain [38]. One review also identified resveratrol and its analogues, powerful antioxidants originally found in grape skins, as activators of TSC1 as a downstream effect through inhibition of AMP kinase, although a more recent manuscript contradicts this assertion [39, 40].

Autophagy Inhibition in Ischemia-Reperfusion Injury

A side-effect of ischemia-reperfusion injury (I/R) is inflammation [41]. In the case of stroke, the loss of blood flow leads to activation of microglia and other pro-inflammatory responses in the brain $[42,43]$. Tetracycline is a well-known and tolerated antibiotic and has been shown to suppress inflammation after stroke [42]. Pre-treatment with tetracycline was shown recently to reduce inflammation by decreasing protein levels of autophagy markers LC3-II and Beclin-1 in a manner similar to 3-MA, although autophagic flux was not addressed, through the inhibition of the NF- $\mathrm{KB}$ pathway, thus leading to neuroprotection from the downstream effects of ischemic stroke [42].

Another drug target identified as relevant to ischemic injury is peroxisome proliferator-activated receptor- $\gamma$ (PPAR $\gamma$ ), because its activation attenuates neuronal apoptosis $[44,45]$. One of the many specific ligands for PPAR $\gamma$ is 15 -deoxy- $\Delta^{12,14}$-prostagladlin $\mathrm{J}_{2}\left(15 \mathrm{~d}-\mathrm{PGJ}_{2}\right)$, which is known as the "anti-inflammatory prostaglandin"[46]. One study found that ischemia-reperfusion injury led to a significant increase in autophagic flux over a 24-hour period, with the highest levels of autophagosome accumulation, LC3-II conversion, Beclin-1, cathepsin-B, and LAMP-1 occurring at the 12-hour time point [47]. Treatment with $15 \mathrm{~d}-\mathrm{PGJ}_{2} 12 \mathrm{~h}$ after $\mathrm{I} / \mathrm{R}$ significantly decreased neural inflammation and substantially reduced the levels of each of the above-mentioned autophagic markers in the area of the injury, in a concentration-dependent manner, and LC3II conversion was verified by 3-MA under the same conditions [47]. While compelling, future studies using 15d$\mathrm{PGJ}_{2}$ would benefit from a time course of its treatment to demonstrate its direct effects on autophagic flux. $15 \mathrm{~d}-\mathrm{PGJ}_{2}$ is currently under investigation as a possible clinical therapeutic agent in a number of areas, and shows potential to alleviate inflammation secondary to ischemic stroke [47].

\section{Neurodegenerative Disease}

\section{Autophagy Enhancement in Neurodegenerative Disease}

Autophagy is relevant to treatment of neurodegenerative disease, as maintenance of its proper function is critical to providing neuroprotection to non-dividing neurons of the brain [48]. Research has been conducted on whether the enhancement of autophagy can protect against neuronal cell loss due to or leading to several neurodegenerative disorders, including Alzheimer's disease, Parkinson's disease and Huntington's disease.

There is mechanistic overlap in the role of autophagy in regulating pathogenesis of different neurodegenerative diseases, and as such, many autophagy-associated therapeutics are being targeted for their potential to treat multiple diseases [48]. Trehalose is a non-reducing disaccharide found in bacteria, yeast, and other invertebrates and acts to potently induce autophagy by an mTOR-independent mechanism $[49,50]$. It has been shown to promote the clearance of both mutant huntingtin (Huntington's disease) 
and $\alpha$-synuclein (Parkinson's disease) [49]. In mouse models of tauopathy (Alzheimer's disease) and prion diseases, trehalose has also been shown to effectively enhance autophagic clearance of the relevant protein aggregates [51-53]. These effects of trehalose are negated in the absence of autophagy machinery, as in the case of ATG5 deficiency, indicating that this effect of trehalose is specific for autophagy [49].

No discussion of autophagy would be complete without an exploration of its enhancement by rapamycin (sirolimus), the small molecule inhibitor that defined the mammalian target of rapamycin (mTOR). Rapamycin has been demonstrated as an effective treatment for cancers and neurodegenerative diseases like Alzheimer's and Parkinson's [54]. As potent inducers of autophagy through the inhibition of mTOR, rapamycin and its derivatives are used to enhance sequestration and degradation of aggregated $\alpha-$ synuclein and amyloid $\beta$, among others [54]. Importantly, rapamycin has been shown to improve neurological function in animal models, such as cognitive/memory deficits in of Alzheimer's disease models, reduction of dopaminergic cell death in Parkinson's disease models, and improvement in motor deficits and decreased neuronal cell death in Huntington's disease models [55]. Importantly, Beclin-1 knockdown has been shown to abrogate neuronal protection by rapamycin, indicating that these protective effects are specific to rapamycin's effects on autophagy [56].

The antidepressant lithium recently underwent phase II clinical trials to assess its efficacy in mediating the clinical outcome in Alzheimer's patients, and has shown some promise as a therapeutic tool [54]. Lithium has been shown to activate autophagy through inositol monophosphatase and glycogen synthase kinase $3 \beta$ (GSK3 $\beta$ ), which enhance autophagy by increasing lysosome numbers, and was able to prevent the hyper-phosphorylation of tau $[57,58]$. It is important to note as well that the effects of lithium on autophagy can be inhibited by 3-MA, thus indicating the specificity of this effect even though lithium has a wide range of activity [57].

\section{Autophagy Inhibition in Neurodegenerative Disease}

Prion diseases are another class of neurodegenerative disease that benefits from autophagy-centric therapy. Similarly to Parkinson's and Alzheimer's, prion diseases result from the aberrant accumulation of protein [51, 59]. Since they are so lethal, have such a rapid onset, and are incurable, it is highly desirable to have effective treatments to help alleviate them. In Creutzfeldt-Jakob Disease (CJD), the buildup of mis-folded prion protein aggregates destroys brain tissue and leads to numerous clinical presentations, including dementia, memory loss, hallucinations, and personality shifts $\left[60^{\bullet}\right]$. One group has developed a screening assay to identify currently approved drugs for alternate use in prion disease therapy. They found that astemizole, a lysosomotropic drug with similarities to chloroquine that is currently in use as an anti-malarial and to treat chronic allergic rhinitis, effectively reduces cell surface prion protein and inhibits prion replication within neuroblastoma cells $\left[60^{\bullet}, 61\right]$. Some mechanistic studies indicate that astemizole stimulates autophagy, as measured by LC3-II conversion, which has been previously shown to clear prions and limit cell-to-cell prion infection [51, 62]. However, this observation could be confounding, as inhibition of autophagosome-lysosome fusion by chloroquine is known to result in an apparent increase in autophagic markers, while the actual function of autophagy, the breakdown and recycling of intracellular components, is in fact inhibited [63].

Astemizole has been shown to be lysosomotropic and to inhibit lysosome function, in a similar fashion to chloroquine [64]. With chloroquine, this results in inhibition of autophagosome-lysosome fusion and a buildup of autophagosomes. Although increased autophagy has been reported to inhibit prion replication and infection, a plausible explanation is that cell surface prions are taken up by endosome-autophagosome mechanisms and held inside infected cells, thus inhibiting infection between cells.

These observations are interesting in that autophagy inhibition is classically considered to be counterproductive in neurodegenerative disease. However, when the impact of pathogenic molecules is considered, this perspective changes. If pathogenic molecules are co-opting autophagic machinery for distribution, either through packaging into exosomal vesicles or dispersal, then inhibiting complete autophagy could help mitigate the process. This has implications for Alzheimer's, Parkinson's, and Huntington's, as there is a growing body of evidence supporting the "infectivity" of pathogenic proteins [59, 65-67]. Alzheimer's disease involves the tau protein that is able to seed neurofibrillary tangles, and it is suspected that $\alpha$-synuclein in Parkinson's disease can be transmitted to neighboring cells through exosome packaging, release, and uptake.

\section{Conclusions}

Researchers are actively working to address the efficacy of exploiting autophagy as a therapy for disease. The sheer extent of research interests is encouraging for the future of autophagy-centric therapeutics. One caution that must be mentioned in closing concerns the definition of autophagy to be used in these investigations. Whether an investigator's working definition includes the entire process of autophagic recycling of material from engulfment to breakdown product release, or if it only includes autophagosome formation 
before lysosomal fusion, or simply LC3-II conversion, this needs to be determined by readers and reviewers prior to analysis of a report. Consistency and agreement within the autophagy research community with respect to interpretation of results will greatly improve the quality and rapidity of effective results being produced.

Acknowledgments The research in this manuscript was supported by the following awards: 1) Veterans Administration Merit Award 1 I01 BX000957-01 (John J. Shacka); 2) National Institutes of Health R01 CA134773 (John J. Shacka); 3) NIGMS MERIT Postdoctoral Fellowship 5 K12 GM088010-05 (Michael P. Nelson).

\section{Compliance with Ethics Guidelines}

Conflict of Interest Michael P. Nelson declares that he has no conflict of interest.

John J. Shacka has received research support from Genzyme Corporation and NIH.

Human and Animal Rights and Informed Consent This article does not contain any studies with human or animal subjects performed by any of the authors.

\section{References}

Recently published papers of particular interest have been highlighted as:

- Of importance

-• Of major importance

1. Oczypok E, Oury T, Chu C (2013) It's a cell-eat-cell world: autophagy and phagocytosis. Am J Pathol 182(3):612-622

2. Symposium on Lysosomes, D.D.C.D.R.A.V.S.C.M.P.C.F. Ciba Foundation Symposium: Lysosomes [proceedings]. 1963; Available from: http://catalog.hathitrust.org/api/volumes/oclc/1749447.html

3. Klionsky D, Codogno P (2013) The mechanism and physiological function of macroautophagy. J Innate Immun 5(5):427-433

4. Li W-w, Li J, Bao J-k (2012) Microautophagy: lesser-known selfeating. Cell Mol Life Sci 69(7):1125-1136

5. Arias E, Cuervo A (2011) Chaperone-mediated autophagy in protein quality control. Curr Opin Cell Biol 23(2):184-189

6. Thomas R, Gustafsson A (2013) Mitochondrial autophagy. Circ J 77(10):2449-2454

7. Christian P, Sacco J, Adeli K (2013) Autophagy: emerging roles in lipid homeostasis and metabolic control. Biochim Biophys Acta 1831(4):819-824

8. Jo E-K et al (2013) Roles of autophagy in elimination of intracellular bacterial pathogens. Frontiers Immunol 4:97

9. Klionsky DJ et al (2008) Guidelines for the use and interpretation of assays for monitoring autophagy in higher eukaryotes. Autophagy 4(2): 151-175

10. $\bullet$ Klionsky D et al (2012) Guidelines for the use and interpretation of assays for monitoring autophagy. Autophagy 8(4):445-544. This publication is especially significant, as it provides useful guidelines developed collaboratively for anyone considering, conducting, or reviewing autophagy research. The author would go as far as suggesting that it be required reading.

11. Hara $T$ et al (2006) Suppression of basal autophagy in neural cells causes neurodegenerative disease in mice. Nature 441(7095): 885-889
12. Gozuacik D, Kimchi A (2007) Autophagy and cell death. Curr Top Dev Biol 78:217-245

13. Levy JM, Thorburn A (2011) Targeting autophagy during cancer therapy to improve clinical outcomes. Pharmacol Ther 131(1): $130-141$

14. Liu H., Z. He, H.-U. Simon (2013) Targeting autophagy as a potential therapeutic approach for melanoma therapy. Seminars in cancer biology

15. Lu S-Z, Harrison-Findik D (2013) Autophagy and cancer. World J Biol Chem 4(3):64-70

16. Motzer R et al (2013) Pazopanib versus sunitinib in metastatic renal-cell carcinoma. N Engl J Med 369(8):722-731

17. Sternberg C et al (2010) Pazopanib in locally advanced or metastatic renal cell carcinoma: results of a randomized phase III trial. J Clin Oncol 28(6):1061-1068

18. van der Graaf W et al (2012) Pazopanib for metastatic soft-tissue sarcoma (PALETTE): a randomised, double-blind, placebo-controlled phase 3 trial. Lancet 379(9829):1879-1886

19. McCann L et al (2010) An indirect comparison analysis of pazopanib versus other agents in metastatic renal cell carcinoma (mRCC). J Clin Oncol 28(15 Suppl):e15128

20. Santoni $\mathrm{M}$ et al (2013) Pazopanib and sunitinib trigger autophagic and non-autophagic death of bladder tumour cells. Br J Cancer. doi: $10.1038 /$ bjc. 2013.420

21. Ha S-D et al (2010) Cathepsin B-mediated autophagy flux facilitates the anthrax toxin receptor 2-mediated delivery of anthrax lethal factor into the cytoplasm. J Biol Chem 285(3):2120-2129

22. Minarini A et al (2013) Synthetic polyamines activating autophagy: effects on cancer cell death. Eur J Med Chem 67C:359-366

23. Kessel D, Vicente M, Reiners J (2006) Initiation of apoptosis and autophagy by photodynamic therapy. Lasers Surg Med 38(5): 482-488

24. Milla Sanabria L et al (2013) Direct and indirect photodynamic therapy effects on the cellular and molecular components of the tumor microenvironment. Biochim Biophys Acta 1835(1):36-45

25. Sparsa A et al (2013) Photodynamic treatment induces cell death by apoptosis or autophagy depending on the melanin content in two B16 melanoma cell lines. Oncol Rep 29(3):1196-1200

26. Monma $\mathrm{H}$ et al (2013) The HSP70 and autophagy inhibitor pifithrin- $\mu$ enhances the antitumor effects of TRAIL on human pancreatic cancer. Mol Cancer Ther 12(4):341-351

27. Fourie A et al (1997) HSP70 binding sites in the tumor suppressor protein p53. J Biol Chem 272(31):19471-19479

28. Leu J et al (2009) A small molecule inhibitor of inducible heat shock protein 70. Mol Cell 36(1):15-27

29. Leu J et al (2011) HSP70 inhibition by the small-molecule 2-phenylethynesulfonamide impairs protein clearance pathways in tumor cells. Mol Cancer Res 9(7):936-947

30. Ranjan K et al (2013) Regulation of HA14-1 mediated oxidative stress, toxic response, and autophagy by curcumin to enhance apoptotic activity in human embryonic kidney cells. BioFactors (Oxford, England). doi:10.1002/biof.1098

31. Aoki $\mathrm{H}$ et al (2007) Evidence that curcumin suppresses the growth of malignant gliomas in vitro and in vivo through induction of autophagy: role of Akt and extracellular signalregulated kinase signaling pathways. Mol Pharmacol 72(1):29-39

32. Demetri $G$ et al (2006) Efficacy and safety of sunitinib in patients with advanced gastrointestinal stromal tumour after failure of imatinib: a randomised controlled trial. Lancet 368(9544):1329-1338

33. Motzer R et al (2007) Sunitinib versus interferon alfa in metastatic renal-cell carcinoma. N Engl J Med 356(2):115-124

34. Gotink K et al (2011) Lysosomal sequestration of sunitinib: a novel mechanism of drug resistance. Clin Cancer Res 17(23): 7337-7346

35. Funk R, Krise J (2012) Cationic amphiphilic drugs cause a marked expansion of apparent lysosomal volume: implications 
for an intracellular distribution-based drug interaction. Mol Pharm 9(5):1384-1395

36. Nadanaciva $\mathrm{S}$ et al (2011) A high content screening assay for identifying lysosomotropic compounds. Toxicol Vitro 25(3): $715-723$

37. Kitagawa K (2012) Ischemic tolerance in the brain: endogenous adaptive machinery against ischemic stress. J Neurosci Res 90(5): 1043-1054

38. Papadakis M et al (2013) Tsc1 (hamartin) confers neuroprotection against ischemia by inducing autophagy. Nat Med 19(3): 351-357

39. Liu M et al (2010) Resveratrol inhibits mTOR signaling by promoting the interaction between mTOR and DEPTOR. J Biol Chem 285(47):36387-36394

40. Li L et al (2013) Resveratrol modulates autophagy and NF- $\kappa B$ activity in a murine model for treating non-alcoholic fatty liver disease. Food Chem Toxicol. doi:10.1016/j.fct.2013.08.036

41. Huang J, Upadhyay U, Tamargo R (2006) Inflammation in stroke and focal cerebral ischemia. Surg Neurol 66(3):232-245

42. Jiang $Y$ et al (2012) Tetracycline inhibits local inflammation induced by cerebral ischemia via modulating autophagy. PloS One. doi:10.1371/journal.pone.0048672

43. Jin R, Yang G, Li G (2010) Inflammatory mechanisms in ischemic stroke: role of inflammatory cells. J Leukoc Biol 87(5): 779-789

44. Lin TN (2005) 15d-Prostaglandin J2 Protects Brain From Ischemia-Reperfusion Injury. Arterioscler Thromb Vasc Biol 26(3): 481-487

45. Wu J-S et al (2009) Ligand-activated peroxisome proliferatoractivated receptor-gamma protects against ischemic cerebral infarction and neuronal apoptosis by 14-3-3 epsilon upregulation. Circulation 119(8):1124-1134

46. Martínez-Gras I et al (2011) The anti-inflammatory prostaglandin 15d-PGJ2 and its nuclear receptor PPARgamma are decreased in schizophrenia. Schizophr Res 128(1-3):15-22

47. Xu F et al (2013) Peroxisome proliferator-activated receptor- $\gamma$ agonist 15d-prostaglandin J2 mediates neuronal autophagy after cerebral ischemia-reperfusion injury. PloS One. doi:10.1371/ journal.pone. 0055080

48. Harris H, Rubinsztein D (2012) Control of autophagy as a therapy for neurodegenerative disease. Nat Rev Neurol 8(2):108-117

49. Sarkar S et al (2007) Trehalose, a novel mTOR-independent autophagy enhancer, accelerates the clearance of mutant huntingtin and alpha-synuclein. J Biol Chem 282(8):5641-5652

50. Chen Q, Haddad G (2004) Role of trehalose phosphate synthase and trehalose during hypoxia: from flies to mammals. J Exp Biol 207(Pt 18):3125-3129

51. Aguib Y et al (2009) Autophagy induction by trehalose counteracts cellular prion infection. Autophagy 5(3):361-369
52. Krüger U et al (2012) Autophagic degradation of tau in primary neurons and its enhancement by trehalose. Neurobiol Aging 33(10):2291-2305

53. Schaeffer V, Goedert M (2012) Stimulation of autophagy is neuroprotective in a mouse model of human tauopathy. Autophagy 8(11):1686-1687

54. Ehrnhoefer D, Wong B, Hayden M (2011) Convergent pathogenic pathways in Alzheimer's and Huntington's diseases: shared targets for drug development. Nat Rev Drug Discovery 10(11): 853-867

55. Wong M (2013) Mammalian target of rapamycin (mTOR) pathways in neurological diseases. Biomed J 36(2):40-50

56. Pan $\mathrm{T}$ et al (2008) Neuroprotection of rapamycin in lactacystininduced neurodegeneration via autophagy enhancement. Neurobiol Dis 32(1):16-25

57. Sarkar S et al (2005) Lithium induces autophagy by inhibiting inositol monophosphatase. J Cell Biol 170(7):1101-1111

58. Parr $\mathrm{C}$ et al (2012) Glycogen synthase kinase 3 inhibition promotes lysosomal biogenesis and autophagic degradation of the amyloid- $\beta$ precursor protein. Mol Cell Biol 32(21):4410-4418

59. Ashe K, Aguzzi A (2013) Prions, prionoids and pathogenic proteins in Alzheimer disease. Prion 7(1):55-59

60. - Karapetyan Y et al (2013) Unique drug screening approach for prion diseases identifies tacrolimus and astemizole as antiprion agents. Proc Natl Acad Sci USA 110(17):7044-7049. This publication, while very specific to prions, provides strong evidence that autophagy inhibition could be beneficial in treatment of neurodegenerative diseases with protein aggregate characteristics.

61. Chong $C$ et al (2006) A clinical drug library screen identifies astemizole as an antimalarial agent. Nat Chem Biol 2(8):415-416

62. Heiseke A et al (2009) Lithium induces clearance of protease resistant prion protein in prion-infected cells by induction of autophagy. J Neurochem 109(1):25-34

63. Shacka J et al (2006) Bafilomycin A1 inhibits chloroquineinduced death of cerebellar granule neurons. Mol Pharmacol 69(4):1125-1136

64. Waterkeyn C et al (1987) Uptake, subcellular distribution and biotransformation of $3 \mathrm{H}$-labelled astemizole in cultured rat hepatocytes. Biochem Pharmacol 36(23):4129-4136

65. Angot E, Brundin P (2009) Dissecting the potential molecular mechanisms underlying alpha-synuclein cell-to-cell transfer in Parkinson's disease. Parkinsonism Relat Disord 15(Suppl 3):7

66. Jucker M, Walker L (2011) Pathogenic protein seeding in Alzheimer disease and other neurodegenerative disorders. Ann Neurol 70(4):532-540

67. Malchiodi-Albedi F et al (2011) Amyloid oligomer neurotoxicity, calcium dysregulation, and lipid rafts. Int J Alzheimer's Dis 2011:906964 